\title{
A produção e a receita pesqueira como indicadores econômicos da pesca artesanal na Amazônia Central
}

\section{Production and income as economic indicators of artisanal fishing in the Central Amazon}

\author{
Maria Angélica de Almeida Corrêa ${ }^{1}$ \\ Sâmea Coelho Bezerra do Nascimento² \\ Daniel Yokoyama Sonoda ${ }^{3}$ \\ Lucirene Souza Aguiar ${ }^{4}$
}

\begin{abstract}
Resumo: A pesca artesanal contribui para a economia da Amazônia com grande impacto na geração de emprego e renda, além da garantia de seguranca alimentar a centenas de famílias ribeirinhas. O município de Manaus, situado na mesorregião Centro Amazonense, é o principal porto de desembarque pesqueiro recebendo a produção capturada de diversos ambientes ao longo da calha Solimões-Amazonas. Dados da produção pesqueira referentes aos anos de 2012 e 2013 possibilitaram avaliar os indicadores de receita e produtividade sobre o desempenho econômico da atividade em termos comparativos. Os indicadores apontaram tendências e suposições acerca da economia da pesca, e sua interação com os aspectos relacionados: ambientais, sociais e mercadológicos, além do entendimento sobre a dinâmica da pesca nos ambientes de onde se origina o pescado desembarcado. A redução na quantidade produzida observada entre os anos foi inferior a 1\%, o que representou cerca de $10 \%$ de dimuinção na receita das pescarias. A variação das espécies desembarcadas, em frequência de ocorrência e volume de produção, explicaram a produtividade e a receita no período, além de indicar a necessidade de estudos e controle sobre quatro categorias de espécies, que responderam por mais de $75 \%$ da produção total. Os indicadores de receita e produtividade se apresentaram como uma ferramenta eficiente para a gestão dos recursos pesqueiros a curto prazo.
\end{abstract}

Palavras-chave: economia da pesca, rendimento, gestão da pesca, manejo.

Abstract: Artisanal fishing contributes to Amazonian economy with great impact on generation of employment and income, besides the food safety guarantee of hundreds of riverside families. Manaus County, located at mesoregion Central Amazonian, it's the main port of fishing landing, receiving captured production of several environment along the Amazonas-Solimões rivers. Fishery production data for the years of 2012 and 2013 made it possible to evaluate indicators of revenue and productivity on economic performance of the activity in comparative terms. The indicators pointed to trends and assumptions about fishery economy

\footnotetext{
${ }^{1}$ Universidade Federal do Amazonas - UFAM - angelicacorrea2011@gmail.com

${ }^{2}$ Centro Universitário do Norte - UNINORTE - scbnascimento@gmail.com

${ }^{3}$ Faculdade Instituto PECEGE - dysonoda@pecege.com

${ }^{4}$ Universidade Federal do Amazonas - UFAM - Iucireneaguiar@gmail.com
} 
and its interactions to related aspects: environmental, social and marketing, besides the understanding on the dynamics of fishing where the fish landed originates. The reduction in the quantity produced between the years was less than $1 \%$, representing close to $10 \%$ decrease on fishery income. Diversity of landed species, frequency of occurrence and production volume, explained the productivity and income in the period, also indicating the need of studies and control over four species categories, answering more than $75 \%$ of total production. Income indicators and productivity presents as an efficient tool for fishery resources management in short time.

Keywords: fishery economy, income, fishing management, management.

\section{Introdução}

A pesca na Amazônia é a atividade econômica de maior impacto para a manutenção da vida das populações tradicionais que utilizam seus recursos, tanto proteicos como fonte de segurança alimentar, quanto financeiros como fonte de renda oriunda de sua produção (Isaac \& Barthem, 1995; Almeida et al., 2006; Barthem \& Goulding, 2007; Lobato, 2009; FAO, 2014). Almeida et al. (2006) estimaram a produção de pescado, contabilizando a quantidade comercializada da ordem de $84 \mathrm{mil} / \mathrm{t} / \mathrm{ano}$, e a quantidade consumida pelos pescadores de subsistência equivalente a $31 \%$ da renda familiar, para dar visibilidade à importância da pesca de pequena escala na região. Além disso, o setor emprega mais de 30 mil pessoas diretamente, e tem capacidade de movimentar a economia em torno de US\$100 a 200 milhões, quando estimada a receita resultante das primeiras negociações entre pescadores e agentes de pesca (Smith, 1985; Ruffino, 1996; Goulding, 1983).

É possível observar outros indicativos a partir dos dados de desembarque, ou produção pesqueira, ocorridos nos principais portos ao longo da calha Solimões-Amazonas, que expressam a dinâmica econômica, ecológica e social da atividade. Batista et al. (2012) analisaram a composição das espécies desembarcadas, por meio dessa dinâmica, e inferiram que cerca de dez categorias respondem entre 70 a $90 \%$ do total desembarcado nos principais portos da Amazônia, apesar da elevada riqueza de suas bacias (Santos et al., 2006). Algumas dessas espécies são as mesmas que sustentam as pescarias por várias décadas, das quais o curimatã (Prochilodus nigricans), o jaraqui (Semaprochilodus spp.), a matrinxã (Brycon amazonicus), o pacu (Mylossoma duriventre), o tambaqui (Colossoma macropomum) e o tucunaré (Cichla monoculus) foram as mais 
relevantes (Santos et al., 2006). Notadamente, a diminuição de alguns estoques pesqueiros, relacionados ao tamanho e abundância das espécies que Ihes eram comuns, é motivo de alerta por especialistas da área perante o poder público e tomadores de decisão, especialmente com relação às espécies de maior valor econômico (Isaac et al., 1996; Petrere Jr., 1983; Bayley \& Petrere Jr., 1989; Barthem \& Goulding, 1997; Isaac et al., 1998; Isaac \& Ruffino, 2000).

Além da pressão pesqueira e da concentração na produção de poucas espécies, as oscilações atípicas, como cheias e secas extremas (Ambrizzi et al., 2007), em decorrência das mudanças climáticas (Marengo et al., 2011; Fearnside, 2012), tem trazido incertezas para a economia da atividade e afetado o seu desempenho. Portanto, o desempenho econômico depende tanto das condições dos ambientes e estoques pesqueiros, quanto das condições de mercado e da eficiência das pescarias (Consultant et al. 2017). Consequentemente, o mau desempenho do setor se reflete na receita líquida e na partilha dos ganhos, ou seja, nos benefícios econômicos objetivados pela tripulação e armadores de pesca, prioritariamente (Prado \& Dremiere, 1990; Cunningham, 1994; Consultant et al. 2017).

A receita das pescarias é um dos indicadores de produtividade e resulta do foco principal que é a subsistência dos pescadores e proprietários de embarcação, assim como na rentabilidade da atividade de uma forma geral (Consultant et al. 2017). Na década de 70, Smith (1969) utilizou, além dos conceitos biológicos, a receita das pescarias, considerando que a duração de uma operação de pesca é proporcional à receita obtida. A receita líquida gera o rendimento percebido, que é proporcional ao ganho econômico geral. Com os indicadores de receita e produtividade é possível promover uma gestão das pescarias que vise melhorias na renda do pescador, desde que elas possam ser alcançadas de forma sustentável (Cunningham, 1994). Além disso, indicam alertas sobre a dinâmica pesqueira em seus diversos aspectos relacionados.

Do ponto de vista do consumidor final, o bom desempenho das pescarias está relacionado ao atendimento à crescente demanda da população, especialmente, aquelas de baixa renda ou em situação de pobreza e estado de vulnerabilidade. Principalmente, quando existe uma elevada relação de dependência pelo pescado, como fonte basilar 
de consumo de proteína animal (Kent, 1997), como no caso da Amazônia. Por outro lado, para as pessoas de classe média e alta, o efeito é possivelmente menor, em função do acesso a outras fontes de proteína animal, sendo pouco afetadas pela "baixa ofertapreços elevados", ou pelo declínio da produção pesqueira.

As pescarias amazônicas envolvem um elevado grau de complexidade que dificulta a obtenção de dados econômicos. Mercados distintos, gosto do consumidor por determinadas espécies, aspectos culturais, ambientais e econômicos, atuação em ambientes de escalas continentais, conformam a dinâmica da pesca de pequena escala na região. As distinções desses aspectos podem ser observadas a cada porto da região. Das seis macrorregiões que representam a calha Solimões-Amazonas, Manaus (AM) é a que concentra maior volume de desembarque (42\%), seguido por Belém (PA) (15\%) e por Tabatinga (AM) (6,5 a 12\%) (Batista et al. 2012). Segundo os autores, a composição das espécies desembarcadas em Manaus tem maior proximidade com as desembarcadas nas macrorregiões do Baixo Solimões e do Alto Solimões, formando um grande grupo representativo sobre a Amazônia.

Com o intuito de gerar informações mais atuais acerca da receita obtida a partir da pesca na Amazônia Central, buscou-se dar enfoque às análises dos dados de desembarque pesqueiro no município de Manaus, Porto Panair, avaliando a produtividade aparente dos ambientes de pesca de origem do pescado, estimando-se a receita das pescarias por mesorregião.

\section{Material e métodos}

O estudo foi realizado na cidade de Manaus (AM), na Feira da Panair, principal porto de desembarque pesqueiro e de comercialização de pescado da região (Figura 1) (Parente \& Batista, 2005; Batista et al., 2012).

Os dados de produção pesqueira foram coletados diariamente, nos anos de 2012 e 2013, no ato do desembarque, envolvendo todos os barcos e pescadores que convergiram suas capturas para a Panair naquele período. A coleta foi realizada por funcionários da Colônia dos Pescadores Z-12, por meio de questionário estruturado de uso deste órgão. Por meio destas entrevistas com os proprietários das embarcações, foram coletados 
Tabela 1. Preço médio das principais espécies desembarcadas no Porto da Panair, Manaus

\begin{tabular}{l|r}
\hline ESPÉCIES & PREÇO MÉDIO \\
\hline acará & 4,25 \\
\hline aracu & 2,96 \\
\hline aruanã & 3,59 \\
\hline bodó & 3,95 \\
\hline branquinha & 2,28 \\
\hline cubiu & 1,57 \\
\hline cuiú-cuiú & 3,58 \\
\hline curimatã & 2,22 \\
\hline dourada & 6,43 \\
\hline fera & 7,36 \\
\hline jaraqui & 3,22 \\
\hline mapará & 3,84 \\
\hline matrinxã & 8,77 \\
\hline pacu & 3,85 \\
\hline pescada & 4,91 \\
\hline piramutaba & 1,14 \\
\hline pirapitinga & 5,89 \\
\hline sardinha & 4,55 \\
\hline tambaqui & 15,85 \\
\hline tucunaré & 5,13 \\
\hline
\end{tabular}

os dados sobre a produção pesqueira: quantidade desembarcada em kg por espécies, por origem (mesorregião e ambiente).

Os preços por pescado foram obtidos pela média simples das informações de fontes de dados secundários nos anos de 2007 (Corrêa, 2009), 2009 (Gandra, 2010) e 2015 (Corrêa, 2017), atualizados pelo Índice Geral de Preços do Mercado (IGP-M) para janeiro de 2018 (Tabela 1).

$$
\dot{p}_{i}=\frac{p_{i 1}+p_{i 2}+P_{i 3}}{3}
$$

Em que:

$p_{i}=$ preço médio da espécie $i$

$p_{i 1}=$ preço da espécie i coletado em 2007, deflacionado para 2018

$p_{i 2}=$ preço da espécie i coletado em 2009, deflacionado para 2018

$p_{i 3}=$ preço da espécie i coletado em 2015, deflacionado para 2018

As pesquisas foram feitas durante a negociação entre pescadores, atravessadores e feirantes em diferentes cidades do estado do Amazonas. 
A receita estimada da pesca foi obtida através do somatório das quantidades de cada espécie por origem multiplicadas por seus respectivos preços médios (Tabela 2 e Tabela 3).

$\sum_{i} p_{i} \cdot q_{i j k}$

Em que: $p_{i}=$ preço médio da espécie $\mathrm{i} q_{i}=$ quantidade da espécie i no ano $\mathrm{k}$

Os dados de desembarque e receita foram organizados por mesorregião (IBGE, 2017), e ambientes de pesca (rios e lagos). As mesorregiões do estado do Amazonas são: Centro Amazonense, Norte Amazonense, Sudoeste Amazonense e Sul Amazonense. A região do Baixo Solimões, que está compreendida na mesorregião Centro Amazonense, em função da sua contribuição pesqueira dentre as demais calhas de rio.

\section{Resultados}

A frota pesqueira que operou nos anos de 2012 e 2013 na capital do Estado do Amazonas, Manaus, foi registrada em 987 e 1149 barcos, que realizaram 6988 e 6991 desembarques, respectivamente. As pescarias operaram em mais de quarenta ambientes, entre rios e lagos, ao longo da calha dos principais rios, Solimões-Amazonas, redefinidas em 4 mesorregiões.

A mesorregião que contribuiu com o maior volume desembarcado foi a Centro Amazonense (Médio Amazonas), com 10.285t que representaram 73\% do total de pescado no ano de 2012. A receita referente a essa região, no mesmo ano, foi estimada em $\mathrm{R} \$$ 41.684.955, equivalente a $68 \%$ do total comercializado (Tabela 2). Os ambientes que se apresentaram mais produtivos foram o Rio Purus (29.72\%), Rio Solimões (22.25\%) e Rio Coari (8,91\%), com uma produção capaz de gerar em torno de 57\% da receita naquele ano (Tabela 02).

Para o ano seguinte, a mesorregião Centro Amazonense voltou a contribuir com o maior volume desembarcado (9.538 t), representando 68\% do total de pescado, porém com um desempenho menor do que no ano anterior. Esta redução nos desembarques se reflete na receita referente a essa região, estimada em $\mathrm{R} \$ 34.753 .347$, correspondente a $63 \%$ do total comercializado. Os ambientes que se apresentaram mais produtivos foram o Rio Purus (33.19\%) e o Rio Solimões (12,51\%), semelhante ao ocorrido no ano 
Tabela 2. Produção pesqueira e estimativa de receita por mesorregião e ambiente, desembarcada no Porto de Manaus, no ano de 2012.

\begin{tabular}{|c|c|c|c|c|c|}
\hline Mesorregião & Ambiente & Produção kg & $\%$ & Receita R\$ & Part \% \\
\hline \multirow{18}{*}{ Centro Amazonense } & RIO PURUS & 4174650 & 29,72 & 17.276 .879 & 28,11 \\
\hline & RIO SOLIMÖES & 3125440 & 22,25 & 12.047 .439 & 19,6 \\
\hline & RIO COARI & 1251700 & 8,91 & 5.688 .148 & 9,26 \\
\hline & RIO NEGRO & 773480 & 5,51 & 3.169 .328 & 5,16 \\
\hline & RIO TAPAUÁ & 404510 & 2,88 & 1.179 .765 & 1,92 \\
\hline & LAGO DO JANAUCÁ & 276100 & 1,97 & 1.241 .902 & 2,02 \\
\hline & RIO AUTAZ & 139800 & 1 & 532.040 & 0,87 \\
\hline & RIO TEFÉ & 46200 & 0,33 & 181.931 & 0,3 \\
\hline & LAGO DOS REIS & 41780 & 0,3 & 142.739 & 0,23 \\
\hline & RIO ARIPUANÃ & 13100 & 0,09 & 54.392 & 0,09 \\
\hline & RIO ARARI & 11230 & 0,08 & 56.555 & 0,09 \\
\hline & LAGO DO MANAQUIRI & 10150 & 0,07 & 50.963 & 0,08 \\
\hline & RIO PRETO DA EVA & 7300 & 0,05 & 28.107 & 0,05 \\
\hline & LAGO COARI & 5200 & 0,04 & 22.330 & 0,04 \\
\hline & RIO ARINI & 2300 & 0,02 & 7.406 & 0,01 \\
\hline & RIO ARANAPU & 1700 & 0,01 & 3.922 & 0,01 \\
\hline & RIO SUPIÁ & 500 & 0 & 1.110 & 0 \\
\hline & subtotal 1 & 10285140 & 73 & 41.684 .955 & 68 \\
\hline \multirow{12}{*}{ Baixo Amazonas } & RIO CANUMÃ & 377000 & 2,68 & 1.924 .324 & 3,13 \\
\hline & RIO B. AMAZONAS & 321850 & 2,29 & 1.239 .804 & 2,02 \\
\hline & RIO BADAJÓS & 287050 & 2,04 & 1.394 .147 & 2,27 \\
\hline & RIO BRANCO & 140990 & 1 & 764.904 & 1,24 \\
\hline & RIO GAMBOA & 117600 & 0,84 & 411.034 & 0,67 \\
\hline & RIO COPEÁ & 116850 & 0,83 & 449.431 & 0,73 \\
\hline & RIO IPIXUNA & 42100 & 0,3 & 135.562 & 0,22 \\
\hline & RIO CICUNDURI & 13950 & 0,1 & 91.628 & 0,15 \\
\hline & RIO CURARI & 3600 & 0,03 & 13.141 & 0,02 \\
\hline & RIO IGAPÓ-AÇÚ & 2300 & 0,02 & 11.139 & 0,02 \\
\hline & RIO CODAJÁS & 200 & 0 & 910 & 0 \\
\hline & subtotal 2 & 1423490 & 10,13 & 6.436 .022 & 10,47 \\
\hline \multirow{8}{*}{ Alto Solimões } & RIO JAPURÁ & 798100 & 5,68 & 6.016 .844 & 9,79 \\
\hline & RIO MADEIRA & 493350 & 3,51 & 1.928 .453 & 3,14 \\
\hline & RIO JURUÁ & 352300 & 2,51 & 1.988 .421 & 3,24 \\
\hline & RIO MAMIÁ & 182480 & 1,3 & 810.322 & 1,32 \\
\hline & RIO JUTAÍ & 120500 & 0,86 & 716.982 & 1,17 \\
\hline & RIO JARY & 43200 & 0,31 & 336.129 & 0,55 \\
\hline & RIO MAMORI & 9800 & 0,07 & 34.770 & 0,06 \\
\hline & subtotal 3 & 1999730 & 14,23 & 11.831 .920 & 19,25 \\
\hline \multirow{5}{*}{ Baixo Solimões } & RIO MANACAPURU & 208350 & 1,48 & 876.528 & 1,43 \\
\hline & RIO PIORINI & 121000 & 0,86 & 538.483 & 0,88 \\
\hline & RIO MARMELO & 3500 & 0,02 & 30.695 & 0,05 \\
\hline & RIO PARAUÁ & 400 & 0 & 2.944 & 0 \\
\hline & subtotal 4 & 333250 & 2,37 & 1.448 .649 & 2,36 \\
\hline \multirow{4}{*}{ Sul Amazonense } & RIO ABACAXI & 4800 & 0,03 & 38.174 & 0,06 \\
\hline & RIO ACARI & 2200 & 0,02 & 13.040 & 0,02 \\
\hline & subtotal 5 & 7000 & 0,05 & 51.214 & 0,08 \\
\hline & TOTAL & 14048610 & 100 & 61.452 .760 & 100 \\
\hline
\end{tabular}


anterior, ambos do Centro Amazonense. Destaca-se o Rio Manacapuru (Baixo Solimões) (6.67\%), conformando os três maiores produtores de pescado com destina ao Porto de Manaus (Tabela 03).

As espécies mais desembarcadas (Tabela 04) variaram nos anos amostrados em volume de produção, mas praticamente compuseram o mesmo grupo, com cerca de vinte espécies ou grupo de espécies. Deste grupo, quatro categorias responderam por mais de $75 \%$ da produção: o jaraqui (Semaprochilodus ssp.), o curimatã (Prochilodus nigricans, Spix \& Agassisz, 1829), o pacú (Myleus ssp., Methynis ssp. e Mylossoma ssp.), a matrinxã (Brycon amazonicus, Spix \& Agassiz, 1829), e a sardinha (Triportheus spp.). As demais espécies que complementaram o desembarque foram: o tucunaré (Cichla spp.), a aruanã (Osteoglossum bicirrhosum, Cuvier, 1829), os grandes bagres registrados pela Colônia de Pescadores como "feras" (Brachyplastistoma filamentosum, Lichtenstein, 1819); (Phractocephalus hemioliopterus, Bloch \& Schneider, 1801), o tambaqui (Colossoma macropomum, Cuvier, 1818), a pirapitinga (Piaractus brachypomus, Cuvier, 1818), a pescada (Plagioscion squamosissimus, Heckel, 1840), e o aracu (Schizodon fasciatus, Spix \& Agassiz, 1829); (Leporinus spp. e Rhytiodus microlepis, Kner,1959).

Os dados de produção por espécie indicaram aumentos e reduções nas quantidades desembarcadas em termos comparativos, contudo, as mesmas continuaram ranqueadas entre as doze mais comercializadas no Porto de Manaus. Essa variação nos volumes de produção pode resultar de muitos aspectos relacionados, dentre eles, ambientais, econômicos, ecológicos, comerciais, que necessitariam de anos de análise. Consequentemente, ela reflete na receita estimada, e pode apontar respostas que expliquem o desenvolvimento do setor nos dois anos amostrados. Comparando os períodos, destaca-se a espécie aracu com o maior incremento no desembarque (65\%), e o decréscimo no volume desembarcado da espécie matrinxã (81\%) (Tabela 05).

Os dados entre os anos analisados indicaram uma redução na produção pesqueira inferior a $1 \%$, que resultou em um impacto de $10 \%$ na receita estimada. $\mathrm{O}$ impacto na receita pode ter ocorrido em função da redução do volume desembarcado de espécies com maior valor econômico no ano de 2013.

Os ambientes de rios foram a principal fonte do pescado desembarcado, e forma- 
Tabela 3. Produção pesqueira e estimativa de receita por mesorregião e ambiente, desembarcada no Porto de Manaus, no ano de 2013.

\begin{tabular}{|c|c|c|c|c|c|}
\hline Mesorregião & Ambiente & Produção kg & $\%$ & Receita $\mathbf{R} \$$ & $\%$ \\
\hline \multirow{15}{*}{ Centro Amazonense } & RIO PURUS & 4.689 .300 & 33,19 & 18.775 .932 & 34 \\
\hline & RIO SOLIMÖES & 1.724 .680 & 12,51 & 5.874 .922 & 10,64 \\
\hline & RIO COARI & 929.080 & 6,57 & 3.165 .607 & 5,73 \\
\hline & LAGO DO JANAUCÁ & 888.420 & 6,29 & 2.447 .368 & 4,43 \\
\hline & RIO TAPAUÁ & 495.150 & 3,5 & 1.703 .553 & 3,08 \\
\hline & RIO NEGRO & 457.820 & 3,24 & 1.617 .924 & 2,93 \\
\hline & LAGO DOS REIS & 125.390 & 0,89 & 345.089 & 0,62 \\
\hline & RIO AUTAZ & 114.400 & 0,81 & 423.392 & 0,77 \\
\hline & RIO TEFÉ & 44.000 & 0,31 & 139.950 & 0,25 \\
\hline & RIO AIAPUA & 30.500 & 0,22 & 98.210 & 0,18 \\
\hline & RIO ARARI & 22.150 & 0,16 & 108.392 & 0,2 \\
\hline & LAGO DO MANAQUIRI & 7.200 & 0,05 & 22.497 & 0,04 \\
\hline & RIO ARIPUANÃ & 6.400 & 0,05 & 20.608 & 0,04 \\
\hline & RIO PRETO DA EVA & 3.200 & 0,02 & 10.304 & 0,02 \\
\hline & Subtotal 1 & 9.537 .690 & 68 & 34.753 .747 & 63 \\
\hline \multirow{12}{*}{ Baixo Amazonas } & RIO CANUMÃ & 368.800 & 2,61 & 1.326 .677 & 2,4 \\
\hline & RIO B. AMAZONAS & 312.500 & 2,21 & 1.101 .919 & 2 \\
\hline & RIO BADAJÓS & 286.250 & 2,03 & 1.027 .355 & 1,86 \\
\hline & RIO BRANCO & 176.950 & 1,25 & 930.634 & 1,69 \\
\hline & RIO GAMBOA & 154.100 & 1,09 & 526.558 & 0,95 \\
\hline & RIO COPEÁ & 77.400 & 0,55 & 251.117 & 0,45 \\
\hline & RIO IPIXUNA & 13.800 & 0,1 & 44.436 & 0,08 \\
\hline & RIO SAPATINI & 12.000 & 0,08 & 38.640 & 0,07 \\
\hline & RIO CURARI & 6.600 & 0,05 & 22.562 & 0,04 \\
\hline & RIO CICUNDURI & 3.900 & 0,03 & 20.871 & 0,04 \\
\hline & RIO IGAPÓ-AÇÚ & 600 & 0 & 2.886 & 0,01 \\
\hline & Subtotal 2 & 1.412 .900 & 10 & 5.293 .653 & 9,59 \\
\hline \multirow{8}{*}{ Alto Solimões } & RIO JAPURÁ & 571.850 & 4,74 & 3.311 .654 & 6 \\
\hline & RIO MADEIRA & 607.300 & 4,3 & 3.656 .059 & 6,62 \\
\hline & RIO JURUÁ & 445.050 & 3,21 & 3.398 .259 & 6,15 \\
\hline & RIO JARY & 163.300 & 1,16 & 558.165 & 1,01 \\
\hline & RIO MAMIÁ & 93.700 & 0,66 & 301.714 & 0,55 \\
\hline & RIO JUTAÍ & 43.800 & 0,31 & 282.293 & 0,51 \\
\hline & RIO MAMORI & 16.300 & 0,12 & 59.042 & 0,11 \\
\hline & Subtotal 3 & 1.941 .300 & 14,49 & 11.567 .186 & 20,95 \\
\hline \multirow{5}{*}{ Baixo Solimões } & RIO MANACAPURU & 942.200 & 6,67 & 3.121 .575 & 5,65 \\
\hline & RIO PIORINI & 119.400 & 0,84 & 390.209 & 0,71 \\
\hline & RIO MARMELO & 18.800 & 0,13 & 60.536 & 0,11 \\
\hline & RIO PUI & 5.000 & 0,04 & 18.989 & 0,03 \\
\hline & Subtotal 4 & 1.085 .400 & 7,68 & 3.591 .309 & 6,5 \\
\hline \multirow{3}{*}{ Sul Amazonense } & RIO ACARI & 3.120 & 0,02 & 17.530 & 0,03 \\
\hline & subtotal5 & 3.120 & 0,02 & 17.530 & 0,03 \\
\hline & TOTAL & 13.980 .410 & 100 & 55.223 .425 & 100 \\
\hline
\end{tabular}


Tabela 4. Produção $(\mathbf{k g})$ e receita estimada das principais espécies desembarcadas por espécie no Porto de Manaus, nos anos de 2012 e 2013.

\begin{tabular}{|c|c|c|c|c|c|}
\hline \multicolumn{3}{|c|}{2012} & \multicolumn{3}{|c|}{2013} \\
\hline ESPÉCIE & PRODUÇÃO kg & RECEITA & ESPÉCIE & PRODUÇÃO kg & RECEITA \\
\hline JARAQUI & $4,355,750$ & $14,025,515$ & JARAQUI & $5,788,490$ & $18,638,938$ \\
\hline MATRINXÃ & $1,433,670$ & $12,573,286$ & PACÚ & $2,094,300$ & $8,063,055$ \\
\hline PACÚ & $2,089,000$ & $8,042,650$ & CURIMATÃ & $2,286,800$ & $5,076,696$ \\
\hline CURIMATÁ & $2,778,560$ & $6,168,403$ & SARDINHA & 738 & $3,357,900$ \\
\hline TUCUNARÉ & 597,89 & $3,067,176$ & FERA & 409,49 & $3,013,846$ \\
\hline FERA & 400,76 & $2,949,594$ & TAMBAQUI & 405,3 & $6,424,006$ \\
\hline SARDINHA & 479,7 & $2,182,635$ & MATRINXÃ & 274,8 & $2,404,866$ \\
\hline TAMBAQUI & 363,25 & $5,757,513$ & TUCUNARÉ & 430,71 & $2,209,542$ \\
\hline PIRAPITINGA & 339 & $1,996,710$ & ARUANA & 477,4 & $1,713,866$ \\
\hline ARUANA & 476,1 & $1,709,199$ & PESCADA & 344,08 & $1,689,433$ \\
\hline OUTRAS & 734,93 & $2,980,080$ & OUTRAS & 731,042 & $2,631,277$ \\
\hline & $14,048,610$ & $61,452,760$ & & $13,980,412$ & $55,223,425$ \\
\hline
\end{tabular}

Tabela 5. Variação da produção das principais espécies desembarcadas no Porto de Manaus, do ano de 2013 para o ano de 2012.

\begin{tabular}{l|l|l}
\hline Nome Popular & Variação naprodução em kg & $\%$ \\
\hline JARAQUI & $1,432,740$ & 33 \\
\hline CURIMATÁ & $-491,76$ & -18 \\
\hline PACÚ & 5,300 & 0 \\
\hline MATRINXÃ & $-1,160,370$ & -81 \\
\hline TUCUNARÉ & $-167,18$ & -28 \\
\hline SARDINHA & 258,300 & 54 \\
\hline ARUANÃ & 1,300 & 0 \\
\hline FERA & 8,730 & 2 \\
\hline PIRAPITINGA & $-124,67$ & -37 \\
\hline PESCADA & 75,170 & 28 \\
\hline ARACU & 128,010 & 65 \\
\hline
\end{tabular}

ram um grupo de seis rios responsáveis por mais de $80 \%$ da produção total no Porto de Manaus, sendo que 50\% dessa produção originada da Mesorregião Amazonense, nos dois anos. No ano de 2012, os rios mais produtivos foram o Purus, o Solimões, o Japurá, o Negro, o Coari, e o Madeira, nessa ordem de importância. No ano de 2013, os rios Manacapuru e Tapauá ficaram entre os seis mais produtivos. O Lago do Janaucá foi o ambiente mais produtivo nessa categoria, nos dois anos de estudo.

\section{Discussão}

A produtividade das pescarias e o bom desempenho econômico, associados à receita e à lucratividade da atividade, estão relacionados à produtividade dos ambientes explorados, valor das espécies explotadas e a duração das pescarias ao longo de um período. Na região Amazônica, essa dinâmica tem forte relação com o regime hidrológico, 
que além das causas naturais, vem sofrendo com efeitos provocados pelas mudanças climáticas (Fearnside, 2012). A frequência e a intensidade dos eventos provocados por esses efeitos podem impactar severamente as produções pesqueiras futuras (Brander, 2007), além dos ambientes com diferentes características, principalmente quando associados à pesca excessiva.

Neste estudo realizado no Porto de Manaus, observou-se que o ano de 2012 foi menos produtivo em relação ao ano de 2013, provavelmente por terem sido marcados por uma cheia extrema e seca atípica, respectivamente. Um maior volume de água como o ocorrido em 2012, pode ter favorecido a dispersão das espécies nos ambientes (Barthem \& Fabré, 2004) dificultando a produtividade das pescarias, enquanto no ano seguinte, com os níveis mais baixos dos rios, as pescarias foram beneficiadas pela concentração dos cardumes nestes ambientes (Pinaya, 2013). Esse ciclo pode explicar possíveis reduções na diversidade e disponibilidade de pescados nos anos seguintes (Mota et al., 2008).

No entanto, apesar das condições as quais os ambientes foram submetidos, a diminuição na quantidade desembarcada não representou diferenças preocupantes. Quanto a receita gerada, houve uma redução de 10\% para o ano de 2013, possivelmente explicada pelo preço das espécies mais desembarcadas naquele ano, de menor valor comercial, em termos comparativos com o ano anterior. A exemplo da espécie matrinxã que apresentou uma baixa produção que impactou a receita estimada, por ser uma espécie muito apreciada e dentre as de maior valor econômico.

Apesar da produção total verificada nos anos testados ser menor do que os já registrados na literatura, em estudos feitos por Souza \& Freitas (2001) sobre uma série histórica de produção do Estado do Amazonas, a mesma não apresentou tendências de crescimento ou de diminuição. Este declínio observado supostamente está relacionado com uma associação entre aumento de esforço, deterioração do ambiente de pesca e maior frequência de eventos extremos afetando os rios da região.

Os ambientes responsáveis pela produção pesqueira, rios e lagos, apresentaram produtividade semelhante nos dois anos, apenas com um decréscimo em torno de $10 \%$ na produtividade do Rio Solimões que, aparentemente, foi compensado pelo melhor de- 
sempenho dos demais ambientes.

Com relação às espécies desembarcadas, o ranque das dez mais produtivas e o volume total também se mantiveram, variando os volumes desembarcados por espécie em comparação com o ano anterior. Neste caso, a manutenção dos níveis de produção parece ter sido compensada quando houve a redução de quatro espécies e o aumento de outras quatro. No entanto, em termos econômicos, é importante observar o valor comercial dessas espécies, para buscar o mesmo equilíbrio no desempenho econômico indicado pela receita da atividade nos anos de estudo.

Um fator que pode explicar o incremento da espécie aracu e a redução da contribuição da matrinxã, é a tendência de substituição progressiva de espécies de maior valor econômico pelas de menor valor, quando aquelas se tornam menos abundantes e mais caras (Santos et al., 2014). Esta substituição temporária possibilita que algumas espécies se recuperem do excesso de esforço de pesca a que são submetidas, e mantém os níveis do total de produção pesqueira para atendimento à demanda crescente na região (Montenegro \& Souza, 2016).

Os ambientes mais produtivos e que mais contribuíram no desembarque do Porto de Manaus estão situados na mesorregião Centro Amazonense. Um dos fatores que contribui para essa eficiência pode ser o fato da capital Manaus estar inserida nela, e ser um grande centro consumidor. Uma das decisões do pescador para aumentar os rendimentos é atuar em áreas que facilitem o escoamento da produção, em sua totalidade, com menores gastos de combustível, rancho, gelo, entre outros insumos de produção (Cardoso et al. 2004). Conciliando esses fatores, estes the conferem melhor retorno financeiro uma vez que ela depende da interação entre fatores de produção e os serviços efetuados com a sua utilização (Faria Jr. \& Batista, 2006). Grandes distâncias entre os locais de pesca e o centro de comercialização elevam os custos de transporte e armazenamento, além de dificultar o acesso ao mercado (Petrere Jr., 1978; Batista, 1998; Parente \& Batista, 2005). Além disso, o Porto de Manaus é considerado o mercado mais atrativo, em termos de demanda e lucratividade, comparado aos demais portos do interior do Estado (Cardoso et al. 2004).

Outro indicativo para explicar a produtividade dessa região é a elevada participa- 
ção dos diversos ambientes, com destaque para o Rio Purus, o Rio Solimões e o Rio Coari (Petrere Jr., 1985; Freitas \& Rivas, 2006; Cardoso et al. 2004; Lopes et al. 2017). Estes rios foram citados como os maiores produtores de pescado na região, e prioritários em estudos pesqueiros devido a sua importância para o setor (Cardoso et al. 2004). Estes sistemas fluviais possuem características biológicas e morfológicas, rios de águas brancas, que favorecem a grande produção de biomassa de peixes, como as áreas de várzea, presentes na sua conformação. Os rios de água branca têm sua produtividade associada a troca de nutrientes com a planície de inundação adjacente, conhecida como áreas de várzea, e dessa forma transportam grande quantidade de sais dissolvidos e sedimentos, enriquecendo-a com nutrientes, resultando em uma elevada produção pesqueira (Barthem \& Goulding, 2007).

A elevada produtividade de três grupos de espécies nos dois anos de estudo (os jaraquis, o curimatã e os pacús), já era esperada uma vez que estes têm destaque nos desembarques da região há várias décadas (Freitas \& Rivas, 2006; Santos et al., 2006; Gandra, 2010; Batista \& Isaac, 2012; Dias-Neto, 2015). As principais espécies das pescarias concordam com as descritas por Freitas \& Rivas (2006), que afirmam que a frota que abastece essa região explora uma grande variedade de espécies de Characiformes migradores, como o jaraqui, o tambaqui, o curtimatã, a matrinxã e os pacús. O jaraqui de escama fina (Semaprochilodus taeniurus, Valenciennes, 1817) juntamente com o jaraqui escama grossa (Semaprochilodus insignis, Jardine \& Schomburgk, 1841), são as principais espécies convergidas para essa região (Freitas \& Rivas, 2006; Santos et al., 2006; Gandra, 2010; Batista \& Isaac, 2012; Dias-Neto, 2015).

Apesar dos jaraquis terem sido as espécies mais desembarcadas no período de 2012-2013, com um aumento de 33\% em relação à produção de um ano para o outro, eles já foram apontados como sobrepescados. Ribeiro (1983) e Batista (1998) observaram que as capturas têm incidido sobre os indivíduos menores, indicando que os estoques estão sendo explorados excessivamente. Ribeiro (1983); Batista (1998) e Freitas (2003) verificaram que apesar dos altos volumes desembarcados em Manaus, a média do comprimento furcal destas espécies tem diminuído nos últimos anos. Com isso, os jaraquis tem sido alvo de constantes negociações entre o poder público e os pescadores, para que sejam inseridas nas listas das espécies protegidas pelo defeso a fim de 
reestabelecer seus estoques. Contudo, uma das justificativas e dificuldades para manejar essas espécies, é por serem consumidas, principalmente, pela população de baixa renda a preços acessíveis (Gandra, 2010).

Os valores de produção registrados para algumas espécies neste trabalho, possivelmente, estão subestimados, como por exemplo, a captura dos grandes bagres, uma vez que essas espécies têm como destino os frigoríficos da região, que possuem uma demanda específica por siluriformes (Schor \& Alves-Gomes, 2010). Os dados sobre a espécie pirarucu (Arapaima gigas) foi suprimida deste trabalho, uma vez que a Colônia não sistematiza a coleta por considerar que sua produção vêm de áreas de reserva. Assim como os dados de produção do tambaqui podem estar sobrestimados, uma vez que alguns desembarques podem ter o pescado oriundo da piscicultura.

O comércio do pescado na Panair ocorre entre meia noite e seis horas da manhã, em função do clima ameno, que evita que o peixe estrague com maior rapidez (Gandra, 2010). As negociações são realizadas por meio de um leilão executado pelos despachantes (Parente \& Batista, 2005). Os preços dos pescados podem variar bastante durante o horário de venda (Gandra, 2010). Da receita obtida dessa operação são descontados os gastos com os insumos, com a manutenção e com a legalização da embarcação; o resultado dessa contabilidade, o lucro bruto, é repartido entre os tripulantes da embarcação por meio de cotas-parte, pré-estabelecidas (Faria Jr \& Batista, 2006). Dessa forma, quanto maior for a receita obtida, maior será o lucro para cada integrante da campanha de pesca. A renda e os custos associados à pesca são fortemente dependentes de variáveis regionais, tais como valor do pescado local e insumos utilizados nas pescarias (Faria-Junior \& Batista, 2006).

Os valores recebidos pelos pescadores são estabelecidos de acordo com a hierarquia e a função na pescaria. Nos estudos executados por Faria Jr \& Batista, (2006), os pescadores recebiam, em média, valores mensais abaixo de um salário mínimo, com exceção da figura do encarregado; os despachantes recebiam rendimentos acumulados de acordo com o número de barcos em que atuavam na comercialização; os armadores de acordo com a quantidade de embarcações que possuíam; e os pescadores de acordo com o número de funções que realizavam na campanha (Cardoso et al., 2004). 
A análise da remuneração obtida pela tripulação é importante por dois motivos: i) costuma ser paga à tripulação de forma proporcional ao rendimento da pesca e, portanto, em longo prazo, o desempenho também é proporcional ao ganho econômico geral; e, ii) a manutenção da renda e emprego contribuem para a subsistência dos agentes de pesca (Consultant et al. 2017). A melhoria do rendimento dos pescadores é frequentemente um objetivo central dos programas de gestão das pescas, especialmente nos países em desenvolvimento, estes devem ser promovidos de forma a serem sustentáveis (Cunningham, 1994).

\section{Conclusões}

Os estudos econômicos são importantes aliados da gestão, disponibilizando parâmetros, teorias e técnicas de estudo relacionados aos fatores de produção das sociedades. Para uma gestão eficaz, os problemas são analisados a partir das questões: o que, quando, quanto e para quem produzir. As respostas vêm das observações, do controle e acompanhamento de comportamentos e tendências, possivelmente representados por indicadores. Os indicadores de produtividade e receita tratados no estudo, apresentaram dados organizados, comparativos e relacionados a variáveis que explicam ou sugerem essas tendências.

Embora os dois anos de dados possam parecer pouco do ponto de vista de uma série histórica, reúnem informações que podem ser utilizadas nas tomadas de decisão, além de fundamentar debates por corroborarem com a vasta, mas desintegrada, literatura sobre a pesca na região, à curto prazo.

A análise de dois anos marcados por eventos de cheia e seca intensa, mostrou que não houve diferenças extremas entre o volume desembarcado de pescado no Porto de Manaus (1\%). Os eventos parecem não ter afetado a produtividade dos principais ambientes de forma significativa, apesar de a Mesorregião Centro Amazonense estar próxima da capital do estado, que supostamente é a mais acessível e sofre maior pressão de pesca. Contudo, algumas espécies indicaram respostas que necessitam de uma análise de correlação mais apurada, em função da redução impactante no segundo ano do estudo. 
Com relação à receita estimada, considerando os preços médios por espécie, a redução foi de $10 \%$. Nesse aspecto, a redução na quantidade de espécies de maior valor econômico teve uma relação direta no percentual da receita estimada, nos levando a analisar dois pontos de vista: 1) as espécies de menor valor compensaram a receita da atividade em detrimento das espécies de maior valor; 2) o aumento do esforço sobre as espécies de menor valor comercial pode trazer consequências aos seus estoques no futuro.

\section{Agradecimentos}

À Colônia dos Pescadores Z-12, Manaus, AM, pela cessão dos dados e atendimento às solicitações do grupo de pesquisa.

\section{Referências}

ALMEIDA, O. T. Manejo da Pesca na Amazônia Brasileira. São Paulo: Editora Petrópolis. 94p. 2006.

AMBRIZZI, T.; ROCHA, R. P.; MARENGO, J. A.; PISNITCHENKO, I.; ALVES, L. M.; FERNANDEZ, J. P. R. Cenários regionalizados de clima no Brasil e América do Sul para o Século XXI: Projeções de clima futuro usando três modelos regionais. Relatório 3. São Paulo: Ministério do Meio Ambiente (MMA), Secretaria de Biodiversidade e Florestas (SBF), Diretoria de Conservação da Biodiversidade (DCBio). 2007.

BARTHEM, R. B.; FABRÉ, N. N. Biologia e diversidade dos recursos pesqueiros na Amazônia. In: M. L. Ruffino (Ed.). A pesca e os recursos pesqueiros na Amazônia Brasileira. p17-62. Manaus: Ed. IBAMA/AM - PROVÁRZEA. 2004.

BARTHEM, R. B.; GOULDING, M. The Catfish Connection: Ecology, Migration and Conservation of Amazon Predators. Columbia University Press, New York, 144p. 1997.

BARTHEM, R.; GOULDIN, M. An unexpected ecosystem: the Amazon as revealed by fisheries. Missouri Botanical Garden Press. 2007.

BATISTA, V. S.; ISAAC, V. J. Peixes e pesca no Solimões - Amazonas: uma avaliação integrada - Brasília: Ibama/ProVárzea. 276p. 2012.

BATISTA, V. S. Distribuição dinâmica da frota e do recurso pesqueiro na Amazônia Central. Tese de Doutorado INPA/UFA. Manaus, Amazonas. 275p. 1998.

BAYLEY, P. B.; PETRERE Jr., M. Amazon fisheries: assessment methods, current status, and management options. (In): Dodge, D.P. (ed.). Proceedings of the International Large River Symposium. Canadian Special Publications, Fisheries an Aquatic Science, 106: p385-398. 1989.

BRANDER, K. M. Global Fish Production and Climate Change, In PNAS, v.4, no 50, p19709-19714. 2007. 
CARDOSO, R. S.; BATISTA, V. D. S.; JÚNIOR, C. H. F.; MARTINS, W. R. Aspectos econômicos e operacionais das viagens da frota pesqueira de Manaus, Amazônia Central. Acta Amazonica. 34(2), p301-307. 2004

CONSULTANT, D. P.; GEE, J.; DIMECH, M. Handbook for fisheries socio-economic sample survey: Principles and practice. Food and Agriculture Organization of the United Nations Technical Officer FAO Fisheries and Aquaculture Department Rome, Italy.136p. 2017.

CORRÊA, M. A. A. Caracterização socioeconômica da pesca comercial no município de Coari, Amazonas. Dissertação de Mestrado. Programa de Pós-Graduação em Ciências Pesqueiras nos Trópicos, da Faculdade de Ciências Agrárias/UFAM, 99p. 2009.

CORRÊA, M. A. A. Subsídios ao ordenamento da pesca de pequena escala na Amazônia: um enfoque econômico / Maria Angélica de Almeida Correa. Tese de Doutorado. Programa de Pós-Graduação em Ciências Pesqueiras nos Trópicos da Faculdade de Ciências Agrárias/UFAM, 131p. 2017.

CUNNINGHAM, S. Fishermen's Incomes and Fisheries Management. Marine Resource Economics. 9:3, p241-252. 1994.

DIAS-NETO, J. O uso da biodiversidade aquática no Brasil: uma avaliação com foco na pesca. Brasília: IBAMA. 288p. 2015.

FAO. Making an income from fisheries. Exploring the Fish Trade. Focus: Fisheries and food security. Fao Newsroom: Data de acesso 17 de março de 2018. Link: http://www.fao.org/focus/e/fisheries/incom.htm. Nov 25. 2014

FARIA-JÚNIOR, C. H.; BATISTA, V. S. Repartição da renda derivada da primeira comercialização do pescado na pesca comercial artesanal que abastece Manaus (Estado do Amazonas, Brasil). Acta Scientarium Human and Social Sciences, 28(1): p131-136. 2006.

FEARNSIDE, P. M. Brazil's Amazon forest in mitigating global warming: unresolved controversies. Climate Policy, v. 12, n. 1, p70-81. 2012.

FREITAS, C. E. C.; RIVAS, A. A. F. A pesca e os recursos pesqueiros na Amazônia Ocidental Fonte: Ciênc. Cult. (São Paulo); 58 (3):p 30-32, 2006.

FREITAS, C. E. C. Levantamento do estado de explotação dos estoques de surubim, jaraqui, tambaqui e curimatã. (In): Forsberg, M, C \& Petrere Jr., (Coord). Estudos Estratégicos, Provárzea/ Setor pesqueiro na Amazônia: análise da situação atual e tendências do desenvolvimento da pesca. Ibama/ProVárzea. Manaus, Amazonas. p76-100. 2003.

GANDRA, A. L. O mercado do pescado da região metropolitana de Manaus. O mercado do pescador nas grandes cidades latino-americanas. CFC/FAO /INFOPESCA. 91p. 2010.

GOULDING, M. Amazonian fisheries. (In): Moran, Emílio F. (Ed.) The dilemma of Amazonian development. Westview Press. p189-210. 1983.

IBGE. Divisão regional do Brasil em regiões geográficas imediatas e regiões geográficas intermediárias: 2017 / IBGE, Coordenação de Geografia. - Rio de Janeiro: IBGE, 2017. 82p. 2017. 
ISAAC, V. J.; BARTHEM, R. B. Os recursos pesqueiros da Amazônia Brasileira. 1995.

ISAAC, V. J.; RUFFINO, M. L.; MCGRATH, D. (In): Search of a New Approach to Fisheries Management in the Middle Amazon Region. Alaska Sea Grant College Program. AKSG98-01. p889-902. 1998.

ISAAC, V. J.; RUFFINO, M. L. Biologia pesqueira do tambaqui, Colossoma macropomum, no Baixo Amazonas. 65-88p. (In): Recursos pesqueiros do Médio Amazonas: Biologia e estatística pesqueira. Coleção meio ambiente. Série Estudos Pesca. 22. Brasília: Edições IBAMA. 350p. 2000.

ISAAC, V. J.; MILSTEIN, A.; RUFFINO, M. L. A pesca artesanal no Baixo Amazonas: Análise multivariada da captura por espécie. Acta Amazônica. 26 (3): p165-208. 1996.

KENT, G. Fisheries, food security, and the poor. Food Policy. v22, p393-404. 1997.

LOPES, G. C. S.; SOUZA, L. A.; INOMATA, S. O. Modelagem das Inter-Relações entre a Pesca e o Regime Fluvial No Rio Purus, AM Rev. Bras. Eng. Pesca 10(2): p94-112, 2017.

LOBATO, A. B. A explotação pesqueira dos grandes bagres migradores na região amazônica e a responsabilidade do estado por dano ambiental transfronteiriço. Dissertação (Direito Ambiental) - Universidade do Estado do Amazonas, Manaus. 148p. 2009.

MARENGO, J. A.; CHOU, S. C.; BETTS, R.; KAY, G.; ALVES, L. M.; TORRES, R.; SANTOS, D.; LYRA, A. A.; SUEIRO, G.; BETTS, R.; CHAGAS, D. J.; GOMES, J. L.; BUSTAMENTE, J. F.; TAVARES, P. Development of regional climate change scenarios (In): South America using the Eta CPTEC/HadCM3 climate change projections: Part 1. Climatology and regional analyses for the Amazon, São Francisco and and the Parana River Basins. Climate Dynamics, Springer-Verlag, 1(1): p1-20. 2011.

MONTENEGRO, L. S.; SOUZA, L. Produção pesqueira e sua relação com as oscilações do ciclo hidrológico e o crescimento demográfico da cidade de Manaus-Am. Scientia Amazonia, 5(2), p14-23. 2016.

MOTA, J. A.; GAZONI, J; GÓES, G. S. Economia das mudanças climáticas. Acesso em 10 de novembro de em 2016. Link: http://www.cetesb.sp.gov.br/wpcontent/uploads/sites/28/2014/05/economia mudanças climaticas.pdf. 2008.

PARENTE, V. M.; BATISTA, V. S. A organização do desembarque e o comércio de pescado na década de 1990 em Manaus, Amazonas. Acta Amazônica. v. 35, n. 3, p. 375-382, 2005.

PETRERE JR., M. A Pesca Comercial no Rio Solimões-Amazonas e seus Afluentes: Análise dos Informes do Pescado Desembarcado no Mercado Municipal de Manaus (1976-1978). Ciência e Cultura, 37, p1987-1999. 1985.

PETRERE Jr., M. Yield per recruit of tambaqui, Colossoma macropomum Cuvier. (In): the Amazonas State, Brazil. Journal of Fish Biology, v.22, p 133-44. 1983.

PINAYA, W. H. A pesca de multiespécies de rede na mesorregião do Baixo Amazonas e suas relações com a variabilidade climática regional e global. Tese de Doutorado. Belém (PA): Universidade Federal do Pará. 228pg. 2013. 
PRADO, J.; DREMIERE P.Y. Fisherman's Workbook. Fishery Industries Division, FAO Food And Agriculture Organization Of The United Nations. Fishing News. Compiled by: Books Oxford. IFREMER, Sete, France. 1. 85p.1990.

RIBEIRO, M. C. L. B. As migrações dos jaraquis (Pisces, Prochilodontidae) no rio Negro, AM, Brasil. Manaus. Dissertação de Mestrado - Inpa/FUA. 192p. 1983.

RUFFINO, M. L. Potencialidades das várzeas para os recursos pesqueiros: uma visão sócio-econômica e ecológica. In I workshop sobre as potencialidades de uso dos ecossistemas de Várzeas da Amazônia p32-53. 1996.

SAMUELSON, P. A. Introdução à análise econômica. 9.ed. Rio de Janeiro: Agir, 683p. 1979.

SANTOS, G. M.; FERREIRA, E. J. G.; ZUANON, J. A. S. Peixes comerciais de Manaus. Manaus: IBAMA/ PróVárzea.146p. 2006.

SANTOS, G. M.; FERREIRA, E.J.G.; VAL, A. L. Recursos pesqueiros e sustentabilidade na Amazônica: Fatos e perspectivas. Hiléia, v5, n. 8.p43-77. 2014.

SCHOR, T.; ALVES-GOMES, J. A. O mercado de bagres e a configuração da rede urbana no alto e médio Solimões, Amazonas, Brasil. p93-110. 2010.

SOUZA, L. A.; FREITAS, C. E. C. Relação entre o pulso de inundação e a produção pesqueira do Estado do Amazonas, Brasil. Revista da UFAM, Ciências Agrárias e Ambientais, Manaus. V. 1 n. 1/2 p 73-86. 2001.

SMITH, N. J. H. The impact of cultural and ecological change on Amazonian fisheries. Biological conservation. v. 32. n. 4. p355-373. 1985.

SMITH, V. L. On models of commercial fishing. J. Pol. Econ., 77: p181-198. 1969. 